\title{
The evolution of episodic-like memory: the importance of biological and ecological constraints
}

\author{
Bas van Woerkum $^{1}$ iD
}

Received: 26 June 2020 / Accepted: 6 February 2021 / Published online: 25 February 2021

(C) The Author(s) 2021

\begin{abstract}
A persisting question in the philosophy of animal minds is which nonhuman animals share our capacity for episodic memory (EM). Many authors address this question by primarily defining EM, trying to capture its seemingly unconstrained flexibility and independence from environmental and bodily constraints. EM is therefore often opposed to clearly context-bound capacities like tracking environmental regularities and forming associations. The problem is that conceptualizing EM in humans first, and then reconstructing how humans evolved this capacity, provides little constraints for understanding the evolution of memory abilities in other species: it defines "genuine" EM as independent from animals' evolved sensorimotor setup and learning abilities. In this paper, I define memory in terms of perceptual learning: remembering means "knowing (better) what to do in later situations because of past experience in similar earlier situations". After that, I explain how episodic memory can likewise be explained in terms of perceptual learning. For this, we should consider that the information in animals' ecological niches is much richer than has hitherto been presumed. Accordingly, instead of asking "given that environmental stimuli provide insufficient information about the cache, what kind of representation does the jay need?" we ask "given that the animal performs in this way, what kind of information is available in the environment?" My aim is not to give a complete alternative explanation of EM; rather, it is to provide conceptual and methodological tools for more zoocentric comparative EM-research.
\end{abstract}

Keywords Animal cognition · Episodic-like memory · Anthropogenic · Biogenic · Anthropocentrism $\cdot$ Zoocentrism $\cdot$ Affordances $\cdot$ Ecological information $\cdot$ Scrub jays

Bas van Woerkum

bas.vanwoerkum@ru.nl

1 Faculty of Philosophy, Theology and Religious Studies, Radboud University, Nijmegen,

The Netherlands 


\section{Introduction}

At first glance - and probably a couple more-cuttlefish, dogs, bees, chimpanzees, mice and rats, pigeons, scrub jays, crows and a lot more species seem to have little in common. Concerning their mental lives, however, at least one striking similarity is purported: A capacity for episodic (or episodic-like) memory (see Dere et al. 2008, Van Horik et al. 2012; Martin-Ordas and Call 2013; and Templer and Hampton 2013 for overviews). The evolutionary function of episodic memory (EM), plausibly, is remembering past situations to anticipate future situations (Suddendorf and Corballis 2007). While anticipation is probably useful for any animal, there is ongoing debate whether animals' anticipatory behavior genuinely relies on episodic memory as opposed to some simpler capacity. Despite diverging claims, then, is episodic memory widespread across the animal kingdom? Do only humans and a handful of other animals have it? Or is it uniquely human?

Most authors have tried to answer this question by primarily defining what episodic memory is in humans and what representational capacities are required for it (Clayton and Dickinson 1998; Dally et al. 2006; Suddendorf and Corballis 2007). Subsequently, they aim to determine whether these representational capacities are present in nonhuman animals. Though major disagreement exists, most authors try to capture the intuitive fact that episodic memories seem decoupled from the environment and can be conjured up at will. EM is therefore often opposed to tracking environmental regularities-a capacity that seems clearly context-dependent and stimulus-bound. This approach-defining EM in humans first, consequently reconstructing how humans evolved this capacity, and to what extent animals did (if at all) - is an anthropogenic method (Keijzer 2017; Lyon and Keijzer 2007, Lyon 2005). Anthropogenic methods are non-ideal for a rich understanding of the evolution of memory abilities, because they define memory independently from animals' evolved biological (i.e. sensorimotor) constitution and their ecological niches. They therefore lack the tools to arrive at a fuller picture of what species share concerning (episodic-like) memory and what is species-specific (see Osvath et al. 2014; Barton 2012; Keijzer 2017). It is no coincidence, then, that most comparisons have been narrowly concerned with answering the question of whether any nonhuman animal has EM either positively or negatively.

The opposite of an anthropogenic approach is a biogenic one. A biogenic approach assumes that "the properties and principles of biological organization present the most productive route to a general understanding of the properties and principles of [...] cognition", where "cognition" is defined as "the processes by which humans and presumably other biological systems come to know the world" (Lyon and Keijzer 2007, p.141). I contend that a consistently evolutionary explanation requires a biogenic method. Accordingly, I define memory in terms of perceptual learning. This entails that remembering means "knowing (better) what to do in later situations because of past experience in similar earlier situations". After that, I explain how EM-which can be loosely defined as memory 
of situations seemingly independent from the present context, and that improve one's dealings with future situations-must likewise be understood in terms of perceptual learning. Note that while sceptics might bring in that a perceptual learning account is not an account of EM (which must by definition be decoupled), the point here is precisely to argue against such an "unconstrained" view of EM. That is, accounts of EM have been conceived of independently from animals' biological, sensorimotor organization and ecological niches. In this regard, a vital point I defend is that while episodic memory may seem unconstrained to us does not imply that is actually is. To make sense of this view, we need to consider that structure and regularity in the environment are indispensable aspects of what makes EM-like abilities possible. While regularity already plays a role in the episodic memory debate, it has been part of the "simpler" explanations. This is because EM seems inexplicable without positing persistent mental representations that mediate between past, present and future. Once representations are invoked, the debate is about the kind of representation: whether it is similarly complex as humans' or different and simpler. The challenge for a biogenic approach, then, is to identify non-trivial environmental regularities that can potentially render representations superfluous.

The biogenic method to be defended here will be developed in terms of affordances and ecological information (Gibson 1979/2015). Affordances are opportunities for action provided by the environment. Ecological information refers to structure in energetic stimulation-for instance, in light waves and air pressure-that allows for perception and action systems (or perceptual systems ${ }^{1}$ ) to detect available affordances. Notably, these systems do not only encompass the sensory organs but also the (active) body and nervous system. For human vision, for example, the eyes are located in the head which sits on a turn-able neck, attached to a moveable body moving in an environment (Gibson 1966). Remembering something, accordingly, means that one has become successful in engaging with specific affordances because prior situations have attuned one's perception and action systems to the relevant ecological information making this engagement possible.

This paper is structured as follows. First, I will briefly describe the evolutionary debate over episodic memories. That is, whether any nonhuman animal has acquired an episodic-like capacity for mental time travel similar to humans (Sect. 2). In Sect. 3, I will propose a biogenic, ecological approach to memory. In Sect. 4, I will argue that remembering distal places and things is possible while staying true to a biogenic method. This requires an understanding of how regularity and structure in the environment makes available ecological information that an animal's perception and action systems can learn to detect, such that much of the work commonly attributed to representations can be "off-loaded" to regularity and structure in the environment. Finally (Sect. 5), I will discuss the questions of evolutionary convergence. My aim is not to account for all details episodic memories can encompass; I instead provide a conceptual analysis that should prove helpful in understanding, in

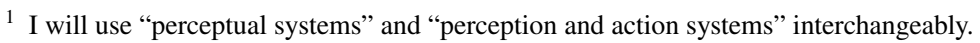


more detail, the similarities and differences between episodic-like memories in various animals.

\section{The evolutionary debate: is episodic memory uniquely human or not?}

Does any nonhuman animal have the capacity to anticipate future events by considering past events, or is this capacity uniquely human? The question has generated a lot of debate. I give a brief sketch of the evolutionary debate; my goal is simply to paint a general picture of the dialectic at work (for more elaborate discussions, see Dere et al. 2008, Van Horik et al. 2012; Templer and Hampton 2013; Martin-Ordas and Call 2013).

As a preliminarily note, the term "episodic-like" memory-prevalent in comparative psychology-denotes uncertainty about whether nonhuman animals have the distinctive phenomenology of reliving personal past episodes of experience characteristic of human EM (Clayton and Dickinson 1998; also called "autonoetic consciousness", see Tulving 1972, 2002). But as several authors have suggested, phenomenology should not be a central part of episodic memory's definition. This is anthropocentric and problematizes the empirical study of EM in nonhuman species (Barrett et al. 2007; Van Horik et al. 2012; Buckner 2013). Instead, the functional and behavioral aspects of EM have guided most research.

An early functional definition of EM was applied to caching behaviour of scrub jays. Scrub jays can cache and recover hundreds of items of food, which they make use of in times of food scarcity (Dally et al. 2006). These foods are often widely distributed and fluctuate in availability. The scrub jay's caching behaviour is a solution to this problem. Comparative psychologists have investigated whether scrub jays may have evolved an ability to flexibly remember the locations of their caches (Clayton et al. 2003; Van Horik et al. 2012). ${ }^{2}$ The authors of these studies defined EM originally in terms of memory for what, where and when: the difference between foods, the different places or locations where they cached the foods, and the different times they buried the foods, respectively (Clayton and Dickson 1998).

Several studies seem to support an EM-like-understood as www-memorycapacity in scrub jays. In well-known studies, this capacity in jays has been examined by making reliance on direct perceptual cues (such as visual or olfactory ones) and association (based on prior learning) impossible. These experiments show that after mere hours have passed, scrub jays first start recovering worms-their preferred food-but will pass over these worms and recover peanuts instead when the worms have gone bad (Clayton and Dickinson 1998; Clayton et al. 2007). Different intervals between caching and recovery were used, so the jays could not learn to rely on this kind of temporal regularity. Other studies ruled out tracking of largerscale environmental cycles. Scrub jays showed the ability to recover foods at several intervals while reliance on astrophysical or geophysical cycles was impossible

\footnotetext{
${ }^{2}$ Something that may also have been true for other species such as cetaceans (Marino, 2002).
} 
(Clayton et al. 2001). Not only environmental, but also internal cues in the form of "memory traces"-neural changes representing associations between stimuli-(see Roberts 2002) have been examined, and discredited: The idea of memory traces is that jays would first recover worms if the memory trace is strong and opt for peanuts if the trace is weak. However, the jays selectively recovered peanuts and worms they cached earlier respectively from two different trays, both of which contained peanuts and worms, but cached at different times (Clayton et al. 2001). Later studies even showed that jays are also capable of taking future motivational states into account by caching more food in the places where they are likely to be hungry (Raby et al. 2007). ${ }^{3}$

Despite the authors' elaborate efforts to prove the contrary, a common response is to claim that these experiments and similar ones can still be explained in simpler terms, often based on the tracking of internal or external regularities, and that for "genuine" EM something more is required. These replies thus question the interpretation of the experiments and often the definition of EM as well.

Well-known opponents of the idea that nonhuman animals have EM are Suddendorf and Corballis $(1997 ; 2007)$. These authors argue that there are different types of memory that vary in their flexibility, of which episodic memory is the most flexible one as it is unconstrained by present circumstances. Episodic memory, moreover, is part of a larger cognitive system of mental time travel (MTT): the adaptive function of episodic memory, they argue, is to anticipate future events, which contributes to survival (see also Dudai and Carruthers 2005a; Suddendorf and Busby 2005). While anticipation seems like an adaptive capacity for any species, relatively inflexible capacities, according to Suddendorf and Corballis (2007) often suffice. What-wherewhen memory, for instance, is not as flexible as MTT: the past could influence the animal's present state and hence a later state, without an intervening episodic memory that is about the past. As the authors themselves put it, often "flexibility extends only to learning to respond to current indicators of upcoming events; behavior is stimulus-bound, or better, bound to perceptual tracking of stimuli. The most important point here, then, is that according to Suddendorf and Corballis (2007) EM or MTT in general is not about tracking regularities but about constructing particularities. Particularity relates to singular episodes of experience, whereas regularities relate to recurring events. Episodic memories, they write, are "decoupled representations" no longer tied to perceptual systems. Such a level of flexibility requires a range of complex capacities, such as metacognition, mental rehearsal, inhibition, recursion and declarative knowledge that no species has of yet convincingly demonstrated. Hence, MTT sets humans apart from nonhuman animals (Suddendorf and Corballis 2007).

A recent, similar defense of the uniqueness of human episodic memory comes from Hoerl and McCormack (2018, 2019). According to these authors, nonhuman animals could act based on a model of the present that is currently updated in a way sensitive to the passing of time (e.g. by systemic use of an "interval timer"),

\footnotetext{
3 A related, interesting case is the chimpanzee Panzee, who could indicate the place where food was hidden outside her enclosure (Menzel 2005; see also Bobrowicz, Johansson \& Osvath 2020).
} 
but that is not itself about the past. Being capable of making past events objects of thought, or put differently, having the ability to locate particular events in objective time, is their mark of decoupling from the present to remember past experience. This requires what they call temporal reasoning abilities (Hoerl and McCormack 2019). While humans represent particular past events objectively and without any obvious restraints, animal behavior can so far be explained in terms of reliance on models of environmental regularities and updating of such models. Thus, Hoerl and McCormack draw a similar conclusion to Suddendorf and Corballis (2007): MTT sets humans apart from other animals. Humans are temporal beings free to navigate through time, while other animals are stuck in the present.

Despite opposing conclusions, these authors all apply a similar "anthropogenic" method (Lyon and Keijzer 2007; Keijzer, 2017): they primarily conceptualize a specific type of experience in humans (i.e. by offering necessary and/or sufficient representational criteria and behavioural indications for this). They then propose an evolutionary hypothesis for how we acquired a capacity that satisfies this conceptual analysis and evaluate to what extent (if at all) nonhuman animals share this capacity with us. Shettleworth (2007) expresses the problems with anthropogenic approaches strikingly: The question "What is mental time travel, and is it unique to humans?' [...] leads to a quest for an existence proof (just one animal with "it" is enough), followed by endless disputes over whether "it" really was demonstrated" (p.332).

Anthropogenic accounts of EM in humans are formulated independently from biological constraints (evolved sensorimotor setup) and ecological constraints (contextual conditions enabling cognitive performances) (see Osvath et al. 2014; Barton 2012; Keijzer 2017, see also Withagen and Van Wermeskerken 2010). As mentioned, the fact that something appears unconstrained does not imply that is unconstrained-why and when we have (and don't have) certain episodic memories is at bottom an empirical question that has not been addressed so far. The main tenet here is that any theory consistent with evolutionary theory should acknowledge constraints on cognition given the body and niche of the animal just as we acknowledge constraints on simple behavior given the body and niche of the animal.

\section{Remembering as knowing better what to do}

The alternative for an anthropogenic method is a biogenic method. According to a biogenic approach, cognition is a biological function for navigating one's niche. Biogenic approaches take "properties and principles of biological organization" as the most fruitful way to understand cognition. In contrast with anthropogenic approaches, biogenic approaches can and do not disregard the material instantiation of cognition. Cognition is seen as an elaboration on basic sensorimotor behavior. This ties into a third point, which is that evolutionary continuity is a presupposition of a biogenic approach. Basic material structures are preserved but become more elaborate, for instance because of the evolution of more elaborate perceptual systems (Lyon and Keijzer 2007). Biogenic methods, finally, are intrinsically zoocentric 
rather than anthropocentric, because they allow for any between-species comparisons and do not prioritize comparison between humans and any nonhuman animal (or nonhuman animals in general).

To keep the capacity to remember firmly grounded in the biological constitution of the animal-which is necessary to understand memory in the context of natural selection - we have to start with a minimal notion of memory and subsequently "work up" in various directions to account for the memory abilities of animals with more elaborate perceptual systems and neural organization (see Barrett 2015). One fruitful way to make this smooth transition from "simple" to more "complex" memory abilities in various niches tenable is based on principles of ecological psychology (Gibson 1979/2015).

How would ecological psychology understand remembering? Ecological psychologists avoid positing mental representations. Instead, remembering is understood in terms of perceptual learning. It means knowing better what to do in later situations due to past experience in similar earlier situations given similar goals (Gibson 1979/2015, p.242, Barrett 2011, p.214, Palatinus and Michaels 2014). Palatinus and Michaels (2014) make a helpful analogy between memory and adaptation, writing that "the consequence of personal experience is not that the old animal has new knowledge, but that it is a new animal that knows better" (p.25). Put differently, earlier experiences do not "accumulate" somewhere and somehow so that they can be conjured up later, but "calibrate" animals' perceptual systems directly, improving their ability to deal adaptively with future situations (see Palatinus and Michaels 2014, p.25).

This description of memory in terms of perceptual learning can be refined by rephrasing it in terms of affordances and ecological information. Affordances are opportunities for action in the environment, while ecological information refers to "the set of structures and regularities" that an animal's perceptual systems automatically detect and "allow an animal to engage with affordances" (Bruineber et al. 2019, 5232). Now, what do affordances and ecological information have to do with remembering? Notice that affordances, as part of their definition, refer both to animals' past abilities and learning trajectories as well as to their future possibilities (Van Dijk et al. 2016; Van Dijk and Rietveld 2018). Hence, if an animal perceives a particular affordance, similar situations in the past have attuned that animal's perceptual systems to the ecological information that indicates that affordance.

A simple example: a jay knows how to efficiently bury a peanut because she has done so before (see Salwiczek et al. 2009). The activity of caching makes the jay's perceptual systems increasingly sensitive to the ecological information that specifies how to do this. Among the informational variables involved in effective caching are optical flow patterns generated by moving the head towards the surface and optical patterns generated by surface transformation as the peanut is inserted. Detecting this information $i$ seing able to successfully bury a peanut. Hence, we have a minimal form of memory, often called procedural memory (Suddendorf and Corballis 2007, p.3): we can say that the jay remembers how to bury the peanut in that later situation if her perceptual systems are attuned to the relevant ecological information.

Take a more complex example now: considering to take a hike in the woods. For this, it is helpful to understand ecological information in terms of ecological 
constraints: if ecological constraints hold between two situation or event types in a niche, instances of the former situation or event provide information about instances of the latter. Ecological information should thus be understood as a relational concept (see Chemero 2009). In this example, the woods afford taking a hike in virtue of the constraints between weather and the forest paths' condition. For instance, if there is heavy rain forest paths are more likely to be swampy. In virtue of this constraint, heavy rain provides information about the swampy forest paths. Accordingly, because too swampy forest paths aren't hike-able for humans, the heavy rain provides information about the forest path's affordance. So, if my perceptual systems have picked up on this information, I perceive that the forest paths are not hike-able, based on my past experience, and decide to stay inside. ${ }^{4}$

Interestingly, because affordances are opportunities for action, perceiving them also implies an anticipatory capacity. I perceive that the forest is not hike-able, but I am simultaneously engaging with a future possibility: whether to go for a hike. Therefore, even if I am sitting at home and considering a hike, detecting heavy rain and low temperature still reliably indicates the affordance, because of those constraints holding between weather conditions and the paths in the woods. It does so less reliably than when I am in the forest where the affordance is fully specified to me - as there could be other conditions that keep the forest path hike-able-but it still does to a certain extent. ${ }^{5}$ Put differently still, the rain and temperature do not exhaustively indicate the affordance (and so I could be wrong), but are nonetheless still pretty reliable indicators. Hence, I am engaging with a distal affordance based on information within my sensory scope (Bruineberg et al. 2019). Accordingly, I know better what to do in this situation now based on similar situations in the past, which allows me to make an adaptive decision and stay inside.

This ability to remember will become fine-tuned with experience; perceptual systems come to detect more fine-grained relations between the ecological information and the hike-ability of the woods (e.g. how the duration of rain relates to the affordance). Jacobs and Michaels (2007) make a helpful analogy with measuring instruments: given that you want to measure a certain environmental property (say air pressure) a barometer will initially be influenced by temperature and humidity and must consequently be "calibrated" to more reliably track air pressure. Similarly, perception and action systems "tune in" on the ecological information specifying affordances.

A few remarks are in place here. First, for an ecological psychologist, memory, perception and anticipation shade off into each other: perceiving is both a kind of

\footnotetext{
4 Note that the word "perception" for ecological psychologists is different than for representationalists. Because for ecological psychologists perception is of affordances, and affordances can be distal, one can perceive what is distal.

5 When ecological information fully specifies or completely reliably indicates an affordances based on ecological laws (e.g. of ecological optics) we speak of "lawful ecological information" or just "ecological information" (Gibson 1979, Stepp \& Turvey 2015). When we detect information that reliably indicates, but does not fully specify affordances we can speak of "general ecological information" (Bruineberg, Chemero \& Rietveld 2019) However, the distinction does not matter too much here, so I will simply use "ecological information" or "information" and speak of more or less reliable information for affordances.
} 
remembering and anticipating, or conversely, both remembering and anticipating are kinds of perceiving (Gibson 1979/2015, 242). Second, on an ecological approach "tracking regularities" in the environment and "remembering" are not understood as different, competing explanations, but as different levels of explanation: Remembering, episodically or not, is done by animals as a whole by engaging with affordances. Tracking environmental regularities - that is, the detection of ecological information-is done by an animal's perceptual systems (see Heft 2010, p.170). Put simply: tracking regularities is a precondition for remembering, not an alternative capacity. It is of course possible to attend to such regularities, but this implies that one is no longer remembering. You can look at the rain and feel the temperature, but to engage with the affordance of the woods - to remember whether it was hike-able-means that this information must somehow be "transparent" to you as a whole person. A similar case is walking on a sidewalk, which means you are not focusing on your feet pressing against the surface and noticing how objects in the distance optically expand as you approach them (both of which will distort the walking).

This ecological definition of memory already entails a rather different perspective on the evolution of memory abilities. To illustrate, Pahl et al. (2007) found that bees can remember what, where and when, but that this ability is circadian-timed meaning that there is a direct cue available. For a representationalist, the question of whether we should call this episodic-like memory-or memory at all-is a real issue. Is the bee really remembering or just acting based on cues in the environment? For an ecological approach, on the contrary, memory is always based on coupling with regularities, though these regularities can be more or less trivial and complex. There is no question of whether the bee is "genuinely" remembering. Circadian rhythm is simply one of the regularities that allows bees to perceive the relevant affordances-that is, to remember situations.

A possible worry at this point is how procedural memory as I characterized itas based on detecting information in environmental regularities — can be "scaled up" to account for episodic-like memory abilities that are "decoupled" from such regularities. Though the hike-example is concerned with remembering distal situations, there are nonetheless direct perceptual cues available (i.e. rain and temperature) so that associative processes could explain my decision to stay inside. Therefore, does remembering particular events not require representations, and hence make it a distinct type of memory from ones based on tracking regularities? In what follows, I will argue that the view on memory I have proposed here can account for EM-like abilities, by recognizing that the ecological information in situations is much richer and wider than has hitherto been presumed.

\section{Remembering and anticipating distal situations}

As discussed in the previous section, ecological constraints between event types offer information about distal affordances. In this part, I argue that we can explain episodic-like memory abilities similarly by considering ecological information and distal affordances. Like procedural memory, EM-like abilities (i.e. remembering situations seemingly independent from the present context, and that improve one's 
dealings with future situations) can be construed in terms of perceptual learning. The main difference between a case like the hiking example and episodic-like memory is not that the former is based on environmental cues whereas the latter is free from such limitations. Both situations involve the environment. Crucially, however, episodic-like remembering involves the tuning of perceptual systems to temporal regularities (whereas the hiking case is mostly concerned with spatial regularities). In this section I will elaborate on this idea, again based on the scrub jay case.

The first thing to note is that events on multiple scales take place in animals' niches. Gibson (1979/2015) wrote that such events are "nested" within one another (p.5). Metaphorically, we could speak of a "clockwork" in the environment, with events taking place on larger and smaller scales, bearings specific relations to each other. There are events taking place over long periods of time extending over years or months, shorter ones taking days, and shorter ones still taking hours, minutes, seconds, or microseconds. Shorter events are nested within longer ones, and multiple shorter events can occur during a single longer one. Such events can be environmental and/or bodily. Among these events for scrub jays, importantly, is the decaying of food items like wax worms (taking about $124 \mathrm{~h}$ ).

Because of this nesting, particular constraints hold between multiple events in scrub jay life. What those events are is an empirical question yet to be addressed. The philosophical point here is that because of such constraints, events within the direct field of view provide ecological information about distal affordances: Just like constraints hold between rain and forest path conditions, such that observing the former offers information about the hike-ability of those forest paths, certain constraints hold between events in scrub jay life and the decay rate of wax worms, such that observing the former events provide information about the recoverability of the worms-even while a jay is spatially and temporally removed from the wax worm. That is: those constraints allow the jay to discover a "temporal invariant": the wax worm's decay rate. Unlike the hiking case, there is no need for events to be causally related to provide information. Events need not change affordances but only indicate it. For reliable indication, multiple events in time and space can and are likely to provide information about distal affordances.

Importantly, note that "observing" or "tracking" events means nothing more than that perceptual systems are detecting the energetic stimulation of that event (e.g. light waves). Accordingly, though human scientists can specify constraints holding between the decaying wax worm event and other events, the jay is not perceiving these when she is trying to recover a worm; the jay is only engaging with affordances. This is similar to the hiking-case: just like the rain and temperature are "transparent" to me in a way that I can perceive whether forests are hike-able, the information available because of constraints between events is transparent to the jay so that she can remember when she buried the worm. Put differently, there is no need for the jay to track and "match" the duration of events first and only then remember that the worm is still edible; instead, remembering that the worm is still edible (or not) $i s$ having your perceptual systems appropriately attuned to the relevant information due to a relevant history of interactions, such that the worms' affordances turn up as relevant for the jay only before it has perished. 
Now, how would this work? How do a jay's perceptual systems attune to the relevant information through a history of interactions? This appears to be partly an evolutionary and partly a developmental story. Part of the "history of interactions" is likely to due to natural selection: Given the irregular distribution of food items in scrub jays' niche and the importance of relying on caches in times of food scarcity, it is likely that over evolutionary time scrub jays' perception and action systems have been predisposed to learn to detect the information relevant with respect to caching and recovery. This predisposition ensures that non-edible foods are avoided most of the time and no useful energy is wasted. This implies that perceptual systems need to selectively attune to information that indicates the most useful affordances (e.g. those that provide most nutritional value). Put differently, given the goal ${ }^{6}$ of recovering, the jays perceptual systems tune in on the information that makes those affordances inviting for the jay that are actually recoverable (see Rietveld and Kiverstein 2014; Rietveld et al. 2018).

The second component of a "relevant history of interactions" is developmental: Over time, the perceptual systems of the jay attune to information that indicates the affordances of caches in increasingly reliable ways - that is, information available in the relation between events. We can again make an analogy with evolution here: just like in natural selection, it is the consequences of behavior that turns out to be adaptive (i.e. successful recovery) that selects for sensitivity (i.e. detection of particular ecological information). So, if a jay recovers a worm while it has gone bad, the perceptual systems are not sufficiently attuned to the information available in the constraints holding between events (or the information was simply not available). What a jay learns, accordingly, is not that a worm perishes in $124 \mathrm{~h}$, but how the worm-perishing event is related to other events that allows the jay to recover the worm when it is still palatable. We should understand what happens in between the moment of caching and recovery, then, not as the retention of the caching episode, but the detection of information by the jay's perceptual systems that in earlier situations have proved to be reliable indicators for the most relevant affordances, given the need for the most valuable food. ${ }^{7}$

Would we call the capacity we discussed "episodic-like memory"? Certainly, the capacity here described is not "decoupled" from the environment and "no longer tied to perceptual systems" (Suddendorf and Corballis 2007). However, it should be clear by now that an ecological account dismisses the possibility of such decoupling, because it ignores biological and ecological constraints. Our memories may seem decoupled, but this does not imply that they are. It is just that the information on which they rely is perhaps less evident and direct and has thus far received little to no attention. Detecting information in the environment about a past caching episode in order to make an adaptive decision (even if that information is non-trivially

\footnotetext{
${ }^{6}$ I use "goal" in a loose sense. I do not take this to mean a mental attitude in the mind of the jay; it indicates selective attunement to information relevant to recovering a particular food, based on natural selection as well as a history of interactions (e.g. having had little to no food recently). That is, the "goal" of the caching system in jays is to detect available food because nutritional levels have become suboptimal.

${ }^{7}$ In the wild, this information is obviously much richer than in experimental contexts, but if the present account is right, we should find such events also in such contexts.
} 
related to the affordance) is undeniably reminiscent of what we commonly gather under "episodic-like memory". The point here is that detecting information in the environment about past events should not be seen as evidence against episodic-like memory, but rather as a methodological principle: we should aim to discover them, for instance through systematic variation (see Reed 1996) in order to understand how and why and when animals are able to remember (and not remember) situations from the past and how this leads them to make adaptive (or non-adaptive) decisions with respect to the future: it is because ecological information is either available or not to the animals' perceptual systems. In this way, an ecological approach could help to account for memory abilities, including EM-like abilities, without starting from the human case that ignores species-specific biological and ecological constraints. ${ }^{8}$ Consequently, we need no longer discuss whether something counts as "genuine" episodic memory.

Two final points that are crucial to evolutionary theory follow naturally from the ecological approach to memory; distinctions that anthropogenic, representationalist approaches have trouble accommodating: individual variation and suboptimality. A first distinction is that between the ecological information available in principle and the information an individual animal picks up at any particular moment. Most of the time, it is not possible to detect all information that a situation can potentially provide. Often, the optimal information is unavailable because environmental conditions are not optimal, or simply because animals are no optimality machines (Withagen and Chemero 2009). Even though much information may be available, an animal may only be able to detect a portion of this. But neither of both are necessary. As Gibson stressed, "Those features of a thing are noticed which distinguish it from other things that it is not-but not all the features that distinguish it from everything that is it not (Gibson 1966b, p.286)" The same likely applies to memory: what is required is sufficient ecological information-information that is reliably enough. However, it is obviously possible that a jay fails to pick up sufficient information and misremember when she cached something. As mentioned, a jay might recover a worm that has gone bad hours before.

A related point concerning the difference between optimal and detected information is that the possibility that all jays detect the same information in similar situations is small for two reasons: because of variability in individuals' perceptual systems, in accord with evolutionary theorizing, and because of different developmental trajectories (Withagen and Chemero 2009). No two animals detect exactly the same information. The crux is that, because the present account "untangles" memory in terms of action and perception, and perceptual systems differ between individuals,

\footnotetext{
${ }^{8}$ Eventually the goal is to account for EM-like abilities in humans all the same. This explanation should capture, just as in any other species, what is species-specific and what is shared with other species. This requires considering the regularities in the human niche-regularities that we have substantially constructed ourselves: we have developed a temporal system using calendars, clocks, holidays and many more things - and we have the language to talk about these things. These are essential in understanding our own memory abilities "from the outside" as it were, rather than based on the intuition that they are "unconstrained" by environmental and bodily factors.
} 
the ecological approach can naturally account for the fact that the ability to remember will differ between individuals based on their sensitivity to information.

My aim in this part has not been to provide a complete account of the (EMlike) memory abilities of jays or other animals in terms of ecological information and affordances. This still requires a lot of empirical and theoretical work, and I am aware that many details have not been offered here simply because we do not know what they are. Nor, in focusing on events, do I want to deny that jays also rely on other mechanisms, such as spatial memory and landmark use (which can further constrain what affordances become relevant. Compare it with having an episodic memory of where you left your keys, where as you consequently go to that place other spatial cues might be available to indicate the affordance even more reliably). Instead, what I tried to do is make steps toward a biogenic method concerning episodic-like memory-especially its temporal component which is arguably the most contested. Instead of asking, "given that environmental stimuli provide insufficient information about the cache, what kind of representation does the jay need?" we ask "given that the animal performs in this way, what kind of information is available in the environment?" This is an important difference: it puts the focus on directly observable aspects of the environment, rather than internal entities that can only indirectly be inferred. This is a method to examine memory abilities from simple to complex while keeping them firmly integrated and consistent with biological and evolutionary constraints, such as the bodies and perceptual systems the animals have reflecting the niches in which the species have evolved. I have tried to show that this is both possible and necessary for a view on the evolution of memory abilities that is consistent with evolutionary theorizing. This is something the "unconstrained" representational capacity of EM arguably does not succeed in. ${ }^{9}$

\section{Evolutionary convergence on remembering}

When authors believe that species have converged on EM-like abilities, what is it that they recognize as being similar? In the beginning of this paper, I suggested that it is the intuition that these behaviors seem to rely on information that cannot be drawn from perceptual cues within the sensory scope. Experiments are devised and conducted to show that various animals either can or cannot remember in this seemingly unconstrained way that humans seem to do. Accordingly, the point of these comparisons is either to look for similiarities between humans and other animals or to show that there is no such similarity, and therefore other animals' capacities are "less" than ours. The question of convergence becomes an all-or-nothing verdict; a one-dimensional hierarchy with a threshold somewhere. This is only possible if cognition and behavior are viewed independently, if EM is thought to operate

\footnotetext{
9 Note that in no way have I denied that animals have specific phenomenological experiences accompanying their remembering abilities; instead, an explanation in terms of regularities in relation between events is, on an ecological account, perfectly compatible with such experiences. The point here has been to account for the functional-behavioral aspects of such abilities from an evolutionary standpoint.
} 
independently from biological sensorimotor constraints. Not only is this dichotomy too simplistic, it lacks an explanation of how an "unconstrained" capacity evolves from a simpler "tracking" capacity (see Osvath and Kabadayi 2019).

In contrast, if we untangle memory in terms of action and perception (Barrett 2011; Penn 2011) we have a clear method to investigate those differences. There will be species-specific differences in memory abilities based on the differences in the animal's ecological niche and the perception and action systems that have evolved to detect the ecological information for perceiving the affordances in these niches. This is an important, largely overlooked observation: we can examine the differences and similarities between species not only because they either fail or succeed in performing the experimental tasks psychologists set for them, but also because of their different bodies and ecological niches: different regularities and relations among such regularities exist in different environments, and animals have evolved perceptual systems to deal with those regularities. Species and even individuals live and have evolved in very differently structured worlds —open and cluttered, aquatic, terrestrial and aerial, large and small scales, to mention a few obvious but major differencesand this will have a big impact on the kind of perceptual systems they have evolved to pick up ecological information.

Now, it may be that animals displaying relatively more flexible behavior have learnt to exploit the nesting of their widely differing niches-the ecological information about distal affordances - in closer-to-optimal ways. It is even possible that many species alive now-even those we commonly dub "simple" such as insectshave evolved the ability to learn to detect information indicating distal affordances, and not only spatially proximal affordances, given that remembering and hence anticipating situations seems useful for most if not all species. Nevertheless, this does not undermine the main message that such capacities are still bound to ecological information available within the sensory scope and that there will be speciesspecific differences in the information that can be and will be detected. Convergence does not have to be all-or-nothing: there can be partial convergence of the perceptual systems to detect particular ecological information in a habitat but also striking differences-differences that could have to do with pre-existing biological constraints (that result from having evolved in different environment) that make "complete" convergence impossible. ${ }^{10}$ In any case, charting both the differences and similarities on the level of ecological information allows for a more detailed taxonomy of the (evolution of) memory abilities than the one we currently employ (see Buckner 2013).

\footnotetext{
10 One can wonder whether two species can "completely" convergence on any ability at all, given their different evolutionary histories and hence different perceptual and motor systems. This is especially true for very distantly related species, like dolphins and ravens whose bodies and perceptual systems could not be more dissimilar. The ecological information in their respective niches is equally different, so can they convergence on abilities at all? However, as I have argued, nesting exists in all niches, and it is interesting to see how resourceful animals are in exploiting this. Convergence on EM-like abilities can be sought, for instance, in the sensitivity to nested regularities across a range of contexts. In any case, what will be important is that we clearly define what we mean by "convergence" (see also Osvath, Kabadayi and Jacobs 2014).
} 
An ecological approach, finally, abandons an anthropocentric perspective in favor of a genuinely zoocentric perspective. This approach provides the tools to investigate the differences between two or more nonhuman species, regardless of what humans can do. While this is something that behavioral ecologists and zoologists, for instance, routineously do, animal cognition researchers often confine themselves to human-nonhuman comparisons. Those ocassional moments when two nonhuman species are compared, this is often because they both share a capacity once thought to be uniquely human (see Barrett 2014). Comparing how bees and ants, or dolphins and whales, or elephants and giraffes, exploit the nesting of their environment to remember situations and anticipate future ones will lead to a much richer comparative psychology.

\section{Conclusion}

In this paper, I have first provided a brief reconstruction of the evolutionary debate about EM: is EM a uniquely human capacity or do some or even many animal share it with us? Anthropogenic approaches define EM in an unconstrained way: they try to capture the intuitive fact that episodic memories are seemingly unbound by present circumstances and can be conjured up at will. This alleged capacity is consequently contrasted with simpler explanations like tracking environmental or internal regularities. I argued that this method is anthropogenic and hard to unite with evolutionary theorizing by making EM-like abilities independent of biological constraints.

In turn, I offered a biogenic approach grounded in ecological psychology. On this view, memory can be seen as improved engagement with affordances, due to an animal's perceptual systems having become attuned to the relevant ecological information indicating those affordances. Memory is a type of perceptual learning. Moreover, I argued that by considering the nested regularities in the environment, remembering distal affordances_-affordances outside the sensory scope-is possible. On this view, EM-like capacities are elaborations and sophistications on basic sensorimotor functioning. Episodic memory, therefore, can also be understood in terms of perceptual learning. In this way, the capacity for episodic memory stays firmly tied to the biological and ecological constraints of animals, as the ability to remember in particular situations will depend on the general ecological information about regularities that animals will be able to pick up. Note, however, that rather than having provided a complete alternative to existing theories of EM, I have provided a method I believe to be both useful and necessary to integrate our knowledge about animal biology and ecology with animal cognition.

In the end, I hope to have contributed to a shift from simplistic yes-or-no-perspectives on EM in nonhuman animals as compared to humans, to a definition that allows for more detailed comparisons between any two species-human or nonhuman. This marks a shift from an anthropocentric to a zoocentric perspective. By having "untangled" memory in terms of perception and action, we have a fruitful method for such comparisons. This is an important insight: differences and similarities between species are not limited to them either passing or failing certain tests, 
but due to their different bodies and ecological niches. Accepting this provides a much stabler ground for future studies into and comparative thinking about memory abilities in species, both closely and distantly related.

Open Access This article is licensed under a Creative Commons Attribution 4.0 International License, which permits use, sharing, adaptation, distribution and reproduction in any medium or format, as long as you give appropriate credit to the original author(s) and the source, provide a link to the Creative Commons licence, and indicate if changes were made. The images or other third party material in this article are included in the article's Creative Commons licence, unless indicated otherwise in a credit line to the material. If material is not included in the article's Creative Commons licence and your intended use is not permitted by statutory regulation or exceeds the permitted use, you will need to obtain permission directly from the copyright holder. To view a copy of this licence, visit http://creativecommons.org/licen ses/by/4.0/.

\section{References}

Barrett L (2011) Beyond the brain: how body and environment shape animal and human minds. Princeton University Press, Princeton

Barrett L (2014) Why dolphins are not aquatic apes. Anim Behav Cognit 1(1):1-18

Barrett L (2015) A better kind of continuity. South J Philos 53(S1):28-49

Barrett L, Henzi P, Rendall D (2007) Social brains, simple minds: does social complexity really require cognitive complexity? In: Emery N, Clayton N, Frith C (eds) Social intelligence: from brain to culture. Oxford University Press, Oxford

Barton RA (2012) Embodied cognitive evolution and the cerebellum. Phil Trans R Soc B 367:20972107. https://doi.org/10.1098/rstb.2012.0112

Bobrowicz K, Johansson M, Osvath M (2020) Great apes selectively retrieve relevant memories to guide action. Sci Rep 10:12603. https://doi.org/10.1038/s41598-020-69607-6

Bruineberg J, Chemero A, Rietveld E (2019) General ecological information supports engagement with affordances for 'higher' cognition. Synthese 196(12):5231-5251. https://doi.org/10.1007/ s11229-018-1716-9

Buckner C (2013) Morgan's Canon, meet Hume's Dictum: avoiding anthropofabulation in cross-species comparisons. Biol Philos 28(5):853-871

Chemero A (2009) Radical embodied cognitive science. MIT Press, Cambridge

Clark A (2008) Supersizing the mind: embodiment, action and cognitive extension. Oxford University Press, Oxford

Clayton NS, Dickinson A (1998) Episodic-like memory during cache recovery by scrub jays. Nature 395(6699):272-274. https://doi.org/10.1038/26216

Clayton NS, Griffiths DP, Emery NJ, Dickinson A (2001) Elements of episodic-like memory in animals. Philos Trans R Soc Lond Ser B Biol Sci 356(1413):1483-1491. https://doi.org/10.1098/ rstb.2001.0947

Clayton NS, Yu K, Dickinson A (2003) Interacting cache memories: evidence for flexible memory use by western scrub-jays (aphelocoma californica). . J Exp Psychology Anim Behav Process 29:14-22. https://doi.org/10.1037//0097-7403.29.1.14

Clayton NS, Salwiczek LH, Dickinson A (2007) Episodic memory. Curr Biol 17(6):R189-R191. https ://doi.org/10.1016/j.cub.2007.01.011

Dally JM, Clayton NS, Emery NJ (2006) The behaviour and evolution of cache protection and pilferage. Anim Behav 72(1):13-23. https://doi.org/10.1015/j.anbehav.2005.08.020

Dere E, Zlomuzica A, Huston JP, De Souza Silva MA (2008) Animal episodic memory. In: E. Dere, A. Easton, L. Nadel, JP. Huston (Eds.) Handbook of Episodic Memory, Elsevier, Amsterdam.

Dudai Y, Carruthers M (2005) Memory: some systems in the brain may better equipped to handle the future than the past. Nature 434:567

Gibson JJ (1966) The senses considered as perceptual systems. Houghton Mifflin, Boston 
Gibson JJ (1979) The ecological approach to visual perception. Psychology Press, NewYork

Heft H (2010) Ecocological psychology in context: James Gibson, Roger Barker, and the legacy of William James's radical empiricims. Psychology Press, New York

Hoerl C, McCormack T (2018) Animal minds in time: the question of episodic memory. In: Andrews $\mathrm{K}$, Beck J (eds) The routledge handbook of philosophy of animal minds. Routledge, New York, pp 56-64

Hoerl C, McCormack T (2019) Thinking in and about time: A dual systems perspective on temporal cognition. Behav Brain Sci 42:1-16. https://doi.org/10.1017/S0140525X18002157

Jacobs DM, Michaels CF (2007) Direct learning. Ecolo psychol 19(4):321-349

Keijzer FA (2017) Evolutionary convergence and biologically embodied cognition. Interface Focus 7:20160123. https://doi.org/10.1098/rsfs.2016.0123

Lyon P, Keijzer FA (2007) The human stain: why cognitivism can't tell us what cognition is and what it does. In: Wallace B (ed) The mind, the world and the body. Imprint Academics, Exeter, pp 132-165

Lyon P (2005) The biogenic approach to cognition. Focus Process 7(1):11-29. https://doi.org/10.1007/ s10339-005-0016-8

Marino L (2002) Convergence of complex cognitive abilities in cetaceans and primates. Brain Behav Evol 59(1-2):21-23. https://doi.org/10.1159/000063731

Martin-Ordas G, Call J (2013) Episodic memory: a comparative approach. Front Behav Neurosci 7:63-63. https://doi.org/10.3389/fnbeh.2013.00063

Menzel C. (2005). Progress in the study of chimpanzee recall and episodic memory. In: HS. Terrace, J. Metcalfe (Eds.), The missing link in cognition: Origins of self-reflective consciousness (p.188-224). Oxford University Press. Oxford. doi:https://doi.org/https://doi.org/10.1093/ acprof:oso/9780195161564.003.0008

Osvath M, Kabadayi C (2019) A theory stuck in evolutionary and historical time. Behav Brain Sci 42:e268. https://doi.org/10.1017/S0140525X19000359

Osvath M, Kabadayi C, Jacobs I (2014) Independent evolution of similar complex cognitive skills: the importance of embodied degrees of freedom. Anim Behav Cognit 1(3):249-264. https://doi. org/10.12966/abc.08.03.2014

Pahl M, Zhu H, Pix W, Tautz J, Zhang S (2007) Circadian timed episodic-like memory-a bee knows what to do when, and also where. J Exp Biol 2010:3559-3567. https://doi.org/10.1242/jeb.00548 8

Palatinus Z, Michaels C (2014) A ten commandments for ecological psychology. In: Shapiro L (ed) The routledge handbook of embodied cognition. Routledge, New York

Penn DC (2011) How folk psychology ruined comparative psychology: and how scrub jays can save it. In: Fischer J (ed) Menzel R. MIT Press. Cambridge, Animal Thinking

Raby C, Alexis D, Dickinson A, Clayton NS (2007) planning for the future by western scrub-jays. Nature 445:919-921. https://doi.org/10.1038/nature05575

Reed E (1996) Encountering the world: toward an ecological psychology. Oxford University Press, Oxford

Rietveld E, Kiverstein J (2014) A rich landscape of affordances. Ecol Psychol 26(4):325-352. https:// doi.org/10.1080/10407413.2014.958035

Rietveld E, Denys D, Westen M (2018) Ecological-enactive cognition as engaging with a field of relevant affordances: the skilled intentionality framework (SIF). In: de Bruin L, Gallagher S, Newen A (eds) The oxford handbook of 4E cognition. Oxford University Press, Oxford

Roberts WA (2002) Are animals stuck in time? Psychol Bull 128(3):473-489. https://doi. org/10.1037/0033-2909.128.3.473

Salwiczek LH, Emery NJ, Schlinger B, Clayton NS (2009) The development of caching and object permanence in western scrub-jays (Aphelocoma californica): Which emerges first? J Comp Psychol 123(3):295-303

Shettleworth SJ (2007) Studying mental states is not a research program for comparative cognition. Behav Brain Sci 30(3):332-333. https://doi.org/10.1017/S0140525X0700218X

Stepp N, Turvey MT (2015) The muddle of anticipation. Ecol Psychol 27(2):103-126. https://doi. org/10.1080/10407413.2015.1027123

Suddendorf T, Busby J (2005) Making decisions with the future in mind: developmental and comparative identification of mental time travel. Learn Motiv 36(2):110-125

Suddendorf T, Corballis MC (2007) The evolution of foresight: what is mental time travel, and is it unique to humans? Behav Brain Sci 30(3):299-313 
Templer VL, Hampton RR (2013) Episodic memory in nonhuman animals. Curr Biol 23(17):R801R806. https://doi.org/10.1016/j.cub.2013.07.016

Tulving E (1972) Episodic and semantic memory. In: Tulving E, Donaldson W (eds) Organization of memory. Academic Press, Oxford

Tulving E (2002) Episodic memory and common sense: how far apart? In: A. Baddeley, JP. Aggleton, MA. Conway (Eds.), Episodic Memory: New Directions in Research. Oxford University Press, Oxford. 269-287

Van Dijk L, Rietveld E (2018) Situated anticipation. Synthese. https://doi.org/10.1007/s11229-01802013-8

Van Dijk L, Withagen R (2016) Temporalizing agency: moving beyond on- and offline cognition. Theory \& Psychology 26(1):5-26. https://doi.org/10.1177/0959354315596080

Van Horik JO, Clayton NS, Emery NJ (2012) Convergent evolution of cognition in corvids, apes and other animals. In: J. Vonk, TK. Shackelford (Eds.), The Oxford Handbook of Comparative Evolutionary Psychology. Oxford University Press. Oxford.

Withagen R, Chemero A (2009) Naturalizing perception: developing the gibsonian approach to perception along evolutionary lines. Theory Psychol 19(3):363-389. https://doi.org/10.1177/09593 54309104159

Withagen R, Van Wermeskerken M (2010) The role of affordances in the evolutionary process reconsidered: a niche construction perspective. Theory Psychol 20(4):489-510

Publisher's Note Springer Nature remains neutral with regard to jurisdictional claims in published maps and institutional affiliations. 